\author{
Mahdi Hussain Al Bandar, Yoon Dae \\ Han, Jamal Al Sabilah, Mohammed \\ Al Suhaimi, Min Soo Cho, HyukHur, \\ Byung Soh Min, Kang Young Lee and \\ Nam Kyu Kim*
}

Department of Surgery, Yonsei University, College of Medicine, Seoul, Korea

Dates: Received: 21 September, 2016; Accepted: 17 October, 2016; Published: 18 October, 2016

*Corresponding author: Nam Kyu Kim, MD, Phd, FRCS, Department of Surgery, Division of Colorectal Surgery, Colorectal Cancer Special Clinic, Yonsei University College of Medicine, Seoul, Korea, 50 Yonsei-ro Seodaemun-gu, Seoul 120-752, Korea, Tel: +82-2-2228-2117; Fax +82-2-313-8289; E-mail: namkyuk@yuhs.ac

www.peertechz.com

ISSN: 2455-2968

Keywords: Splenic flexure; Left colon cancer Vessel ligation; Optimum level

\section{Research Article \\ Optimum Level of Vessel Ligation in Splenic Flexure Cancer}

\section{Introduction}

Splenic flexure carcinoma (SFC) incidence is very low in colorectal cancer (CRC) that has been reported in 2 to $8 \%$ of the total colonic cancer. Often time presented in an advanced stage with high risk of obstruction that contributed in poor prognosis [1,2]. In addition, some other studies mentioned about the rate of SFC obstruction that accounted four times greater than other colon cancer sites [3], and that's due to late presentation and insidious onset of the disease. Poor prognostic features of SFC could reflect the behavior of tumor biology and characteristics of SFC, which are poorly defined. Therefore, extensive surgical resection has to be addressed well in order to achieve better results. Basically, Excision of the tumor along vascular supply and its lymphatic drainage accounted for bottom line to reduce the chance of local recurrence in CRC [4].

Japanese Society for Cancer of the Colon and Rectum rules [5], emphasizing the policy of oncology safety. They stated the necessity of tumor dissection at its root as well as separation of meso-colon fascia from adherent retropretoneal fascia. Subsequently, more lymph node would be anticipated. Therefore, the determination of the tumor-related supplying arteries is critical for deciding the range of lymph node dissection in a certain tumor location. In 1995, Toyota et al. [6], published rationale for extent of lymph node dissection for right colon cancer, which subsequently became one of the main bases for deciding the range of lymph node dissection in Hohenberger's proposal for right sided colon cancer. He emphasized unique concept at which anatomical fascia planes should be followed to yield high number of metastatic lymph node and named it complete mesocolon excision (CME) [7]. CME technique contributed to enhance overall survival and reduce local recurrent rate dramatically from $82.1 \%$ to $89.1 \%$ and $6.5 \%$ to $3.6 \%$, respectively [7]. Bokey et al. [8] showed enhanced overall survival up to $15.6 \%$ when dissection take place in embryological planes. Moreover, central venous ligation and division of feeding vessel at the root have shown a successful concept to retrieved higher number of lymph node metastasis [8]. On top of that, higher number of lymph node harvested attributed in accurate staging [9]. CME principles for right-sided resection equivalent to the current TME principles for left sided resection.

Therefore, optimal vessel ligation has been discussed and studied well in the right-sided colon cancer with anticipated success to achieve oncological benefits. However, guideline to determine the level of vessel ligation in SPC is still remained in debates. The concept of high vessel ligation is to include resection of the paracolic nodes, intermediate nodes and apical lymphnodes, which defined as D3 lymph-adenectomy. D3 lymph-adenectomy have been widely used and accepted in Japan, China and Korea [10-12]. In case of SFC, whether high level of vessel ligation is required to include LMC artery or not, yet has not been proposed. To our knowledge, this is the first study aiming to investigate the adequacy of vessel ligation in SFC in term of overall survival (OS) and local recurrence.

\section{Materials and Methods}

\section{Patients}

Throughout 2005 until 2012 records were reviewed and analyzed retrospectively.170 Patients diagnosed with distal transverse colon cancer operated by left colectomy were investigated. Follow up charts and management had been prospectively collected. Further analysis performed and 43 patients histologically proven adenocarcinoma of SFC were enrolled in our study. They were allocated according to the level of vessel ligation (LCA plus MMC) marked as group (A) vs. (LCA plus LMA) as group (B). Level of vessel ligation was 
completely decided according to surgeon's preferences due to the lack of standard guidelines. Therefore, faculty members have made an effort to determine the level of vessel ligation by their own experience.

Patient's characteristics, peri-operative clinical results, and longterm oncologic outcomes were compared and matched between two groups as shown in Table 1. Exclusion criteria were old age more than 85 year old, synchronous primary tumor, stage VI disease, clinically unfit for surgery, American Society of Anesthesiologists (ASA) IIIIV, tumor involved adjacent organ and non-complaint patients, and otherwise all other patients were included. For each patient data including gender, age, body mass index (BMI), conversion to laparotomy, intra-operative complications, post-operative complications, number of excised lymph nodes, readmission, and reoperation rates were studied. In addition, 4-6 cycles of adjuvant chemotherapy (ACT) were given to whom diagnosed with adenocarcinoma with tumor extend beyond T1b or deeper, particularly for those cannot tolerate general anesthesia, unfit for definitive operation or according to patients request and selecting for wait and see approach with close monitoring. Data were prospectively recorded in a CRC database and retrospectively analyzed.

\section{Diagnostic staging and postoperative care}

Colonoscopy, biopsy and computed tomography (CT) scan were used for diagnosis in all patients. The American Joint Committee on Cancer (AJCC) $7^{\text {th }}$ edition classification and stage groupings were used for tumor assessment. Staging of the tumor achieved by chest $\mathrm{X}$-ray, chest and abdomen CT scan as well as liver MRI in case of suspected liver metastasis. Tumor was successfully localized by either colonoscopy tattooing with Indian ink or intra-operative colonoscopy marking in case of early CRC.

Patients monitored postoperatively in a uniform protocol among our institution stuffs and oncology department. Patients managed and closely observed in the floor by an expert physician assistance and full team of surgeons on duty. In term of feeding after surgery, sips of water will be allowed in 24 hours after surgery then fluid diet

\begin{tabular}{|l|l|l|l|}
\hline \multicolumn{3}{|l|}{ Table 1: Patient's Characteristics. } \\
\hline Variables & Group A (n=24) & Group B (n=19) & P-Value \\
\hline Age & $63(40-82)$ & $66(37-83)$ & 0.721 \\
\hline Sex & & & 0.302 \\
\hline Male & 17 & 16 & \\
\hline Female & 7 & 3 & \\
\hline BMI & $23.1 \pm 2.9$ & $23.4 \pm 2.6$ & 0.689 \\
\hline Op method & & & 0.003 \\
\hline Open & 9 & 0 & \\
\hline MIS & 15 & 19 & \\
\hline Preop. CEA & $3.6 \pm 4.6$ & $5.2 \pm 8.5$ & 0.424 \\
\hline Postop. CEA & $1.6 \pm 1.7$ & $1.8 \pm 1.2$ & 0.769 \\
\hline Abbreviation & & A: & \\
\hline
\end{tabular}

Abbreviations: Group A: ligation of left colic artery + marginal branch of middle colic artery (LCA +MMC) marked as group (A), Group B: ligation of left colic artery + left branch of middle colic artery (LCA+LMA) marked as group (B), BMI: body mass index, Postop:post-operative,Preop:pre-operative, CEA: Carcinoemberyonic Antigen, OP: operation, MIS: minimum invasive surgery. will follow in the next 48 hours after surgery. Abdominal drains usually removed at 5 th day postoperatively if there was no suspicious bleeding or abdominal collections. As long as patients tolerated orally, passed flatus and declared smooth postoperative recovery, can be discharged home and followed up in out patient's clinic within 2 weeks' time then every 3 months for the first year and every 6 months for the first 2 years and then annually thereafter until death or until they were lost to follow-up. In accordance with common practice at our institute, a clinical diagnosis was considered a sufficient evidence of recurrence; however, radiological or endoscopic confirmation was essential. In order to maximize our care, each visit to our out patients clinic, we routinely do clinical examinations, common blood chemistry $(\mathrm{CBC})$ tests, serum CEA level, chest X-ray and abdominal and pelvic CT if needed as well as annual colonoscopy to rule out any possible recurrent or developing secondary tumor. Other imaging modalities (CT, MRI and bone scans) requested in case of recurrence or metastases were anticipated.

\section{Technique}

Five expert surgeons in the field of CRC with an experience of more than 200 procedures were selected to join our study criteria. This Study conducted in a very highly sophisticated setting in joint commission international (JCI) approved oncology center. Before 2007, procedures were performed by exploratory laparatomy but we changed our practice to laparoscopic surgery afterward. All patients routinely received mechanical bowel preparation. Low molecular weight heparin for deep venous thrombosis prophylaxis was used routinely. Antibiotic prophylaxis with cefuroxime was given 30 minutes before anesthesia induction and two doses postoperatively. All patients placed in a low lithotomy position, protected by straps for legs and pneumatic device to prevent deep venous thrombosis. In laparoscopic operation, five ports placed trans-abdominally, general inspection to rule out immediate complications or distant metastasis deposits. All our procedures started from medial to lateral approach with oncological concept or radical tumor resection, complete lymphadenectomy and central ligation of the feeding vessels. In addition, no touch technique is a well-known approach in our institute at which crucial tumor dissection and tumor extraction through abdominal wall by using Alexis retractor in order to prevent tumor contact to skin. Procedure end with two drains placed in the abdomen cavity to monitor perioperative complication probabilities that removed at 5th day postoperatively. Likewise in open surgery, all oncology principles and safety were as identical as in laparoscopic group except cosmoses part where longer incision expected in open surgery, which usually located at midline of the abdomen and extended longer as per necessary.

\section{Study definitions}

OS was defined as the time from surgery to death from any cause. DSS was defined as the time from surgery to death related to cancer. DFS was defined as the time from surgery to any recurrence. Postoperative complications were grouped according to the time of the event; any adverse events occurring within 30 days. Splenic flexure cancer was defined as a tumor located in the distal third of the transverse colon, or in the left colonic angle, or in the proximal descending colon within $10 \mathrm{~cm}$ from splenic flexure [13]. Recurrent 
is identified if any mass or nodule had shown in the imaging studies during follow up time. In term of technical part, level of vessel ligation confirmed by expert surgeons and reassert by gross examination of the specimen.

\section{Statistical analysis}

Data are summarized as frequencies and percentages for categorical variables. Medians and ranges are used for continuous variables. Chi-squared tests were used to compare proportions and independent $t$ test was performed for comparison of continuous variables. Uni-variable analysis of OS and DFS were carried out by the Kaplan-Meier method. All results were considered statistically significant if $\mathrm{p}$ value was less than 0.05. Statistical Package for the Social Sciences for Windows (Version 20.0, Chicago, IL) was used for data analyzing.

\section{Results}

\section{Patient characteristics}

From early 2005 till the end of 2012, forty-three patients diagnosed with SFC enrolled in our data analysis. Majority of SFC patients in our institute were men, accounting for 33 patients (76.7\%), 10 patients (23.3\%) were women. The mean age was 66 year old, ranging between (37-83years). Patient's weight were almost identical in both groups with BMI average of $(23.1 \pm 2.9$ vs. 23.4 $\pm 2.6, \mathrm{p}=0.69$ ) in-group $\mathrm{A}$ and $\mathrm{B}$, respectively. CEA marker was not significantly changed post-operatively, as it was within normal range preoperatively at an average of 3.6- $4.6 \mu \mathrm{g} / \mathrm{ml}$. All our patients were operated with a curative intent. Minimum invasive surgery started at early 2007 in our institute that we had few patients operated by exploratory laparotomy, 9 patients $(21 \%)$ vs. 34 patients $(79 \%)$, $\mathrm{p}=0.003$, respectively as demonstrated in Table 1 .

\section{Tumor stage and histological finding chemotherapy course}

There were no significant different in tumor stage between both group. Stage 1 rated at $25 \%$ in group A vs. $26.31 \%$ in group B, stage 2 and 3 found $33.4 \%, 4.2 \%$ vs. $42.1 \%, 31.57 \%, p=0.77$, respectively. Both arms received resemble course of ACT, afloropyrimidine-based regimen. There was no neo-adjuvent therapy had given to our patients. 15 patients received ACT in group A whereas 8 patients received ACT in group $B$, without statistical significant difference between two arms of the study as shown in Table 2. The mean longest diameter of the tumors was $5.4 \pm 4$ in group $\mathrm{A}$ and $4.7 \pm 2.1$ in group $\mathrm{B}, \mathrm{p}=0.469$. According to the cell differentiation, majority of the tumor grade turned out to be moderately differentiated cancer, rated at 19 patients (79.1\%) in group A and 15 patients (69.9\%) in group B. Few other patients found to be well or poorly differentiated cell tumors. Overall $\mathrm{p}$ values were insignificant in regards tumor cell differentiation in both groups, $\mathrm{p}=0.63$. Incidence of lympho-vascular invasion reached only $6(25 \%)$ vs. 3 patients (15.78\%), p=0.461, in A and B group, respectively. Tumor clearance achieved successfully in both arms in term of proximal margin achieved at $14.1 \pm 6.5 \mathrm{~cm}$ in groups $\mathrm{B}$ and 8.8 $\pm 3.9 \mathrm{~cm}$ in group $\mathrm{A}, \mathrm{p}=0.006$. Distal margin reported at $11 \pm 7.4 \mathrm{~cm}$ and $9.8 \pm 4.7 \mathrm{~cm}, \mathrm{p}=0.58$, respectively. Total lymph node harvested was oncologically safe in both groups at an average of $19 \pm 10$ lymph nodes in group A and $15 \pm 6.7$ lymph nodes in group B. As well as the rate of positive lymph nodes retrieved in our patients were at $1.6 \pm 4$ and 1.4 $\pm 2.7, \mathrm{p}=0.882$, respectively, as shown in Table 3,3A.

\section{Overall survival and local recurrent rate}

Local recurrence reported in 4 patients $(16.6 \%)$ at group A and only 2 patients (10.5\%) found in group B but had not reached statistical significant difference between both groups $(\mathrm{p}=0.56)$.DFS analyzed at 79.1 month vs. 84.7 month respectively with $95 \%$ confident interval at (67.4- 90.8) in group A and (73.4 - 96) in group B. overall survival

\begin{tabular}{|l|l|l|l|}
\hline \multicolumn{4}{|l|}{ Table 2: Tumor Stage, Recurrent and Chemotherapy Treatment. } \\
\hline Tumor & Group A (n) & Group B (n) & P-Value \\
\hline Stage & & & 0.772 \\
\hline 1 & 6 & 5 & \\
\hline 2 & 8 & 8 & \\
\hline 3 & 10 & 6 & \\
\hline Recur. & & & 0.564 \\
\hline Yes & $4(16.66 \%)$ & $2(10.5 \%)$ & \\
\hline No & 20 & 17 & \\
\hline Adj. Therapy & & & 0.183 \\
\hline Yes & 15 & 8 & \\
\hline No & 9 & 11 & \\
\hline Abbreviations: & Adj: & & \\
\hline
\end{tabular}

Abbreviations: Adj: adjuvant chemotherapy, Group A: ligation of left colic artery + marginal branch of middle colic artery (LCA +MMC) marked as group (A), Group B: ligation of left colic artery + left branch of middle colic artery (LCA+LMA) marked as group (B), Recur: recurrence,

Table 3: Histological Outcome between two groups in Splenic Flexure Cancer 3A: Radical Margins and Harvested Lymph Node.

\begin{tabular}{|l|l|l|l|}
\hline Variables & Group A $(\mathbf{c m})$ & Group B $(\mathbf{c m})$ & P-Value \\
\hline P margin & $8.8 \pm 3.9$ & $14.1 \pm 6.5$ & 0.006 \\
\hline D margin & $9.8 \pm 4.7$ & $11 \pm 7.4$ & 0.583 \\
\hline Total LN (n) & $19 \pm 10$ & $15 \pm 6.7$ & 0.171 \\
\hline Positive LN (n) & $1.6 \pm 4$ & $1.4 \pm 2.7$ & 0.882 \\
\hline Tumor size & $5.4 \pm 4$ & $4.7 \pm 2.1$ & 0.469 \\
\hline
\end{tabular}

Table 3A: Cell Differentiations and Cellular Invasion.

\begin{tabular}{|l|l|l|l|}
\hline Variables & Group A $\mathbf{n}(\%)$ & Group B $\mathbf{n}(\%)$ & P- Value \\
\hline CD & & & 0.637 \\
\hline WD & $4(16.66)$ & $2(10.5)$ & \\
\hline MD & $19(79.1)$ & $15(69.9)$ & \\
\hline PD & $1(4.1)$ & $2(10.5)$ & \\
\hline LVI & & & 0.461 \\
\hline Yes & $6(25)$ & $3(15.78)$ & \\
\hline No & $18(75)$ & $16(84.2)$ & \\
\hline
\end{tabular}

Abbreviations: A: ligation of left colic artery + marginal branch of middle colic artery (LCA +MMC) marked as group (A), Group B: ligation of left colic artery + left branch of middle colic artery (LCA+LMA) marked as group (B), CD: cell differentiations,cm: centimeter, D: Distal, LN: lymph node, LVI: Iympho-vascular invasion, P: proximal, WD: well differentiated, MD: moderate differentiated, PD: poor differentiated, n: number. 
rated at 75.2 month vs. 79.1 month with 95\% confident interval (64.1$86.24)$ and (64.3 -93.9) respectively, which haven't shown statistical differences between both groups $(\mathrm{p}>0.05)$ as shown in Figure 1. There were no death-related, immediate postoperative complications (within1 month of surgery). No peri-operative complications were recorded for both study groups. No conversion case was reported in our entire patients group.

\section{Discussion}

Surgical management of SFC is varies due to variability and limitation of blood supply to the splenic flexure area, which make troublesome in taking decision in regards level of vessel ligation. Decision should weight benefits of oncology safety by higher ligation of feeding vessel vs. the risk of comprising the blood supply to the anastomosis site that could resulted in higher incidence of leak or chronic stricture. This concern has been varies among surgeons and institutes. Interestingly, Sadler et al. [14], advocated extended right colectomy an option for SFC. He believed that lymph nodes metastasis

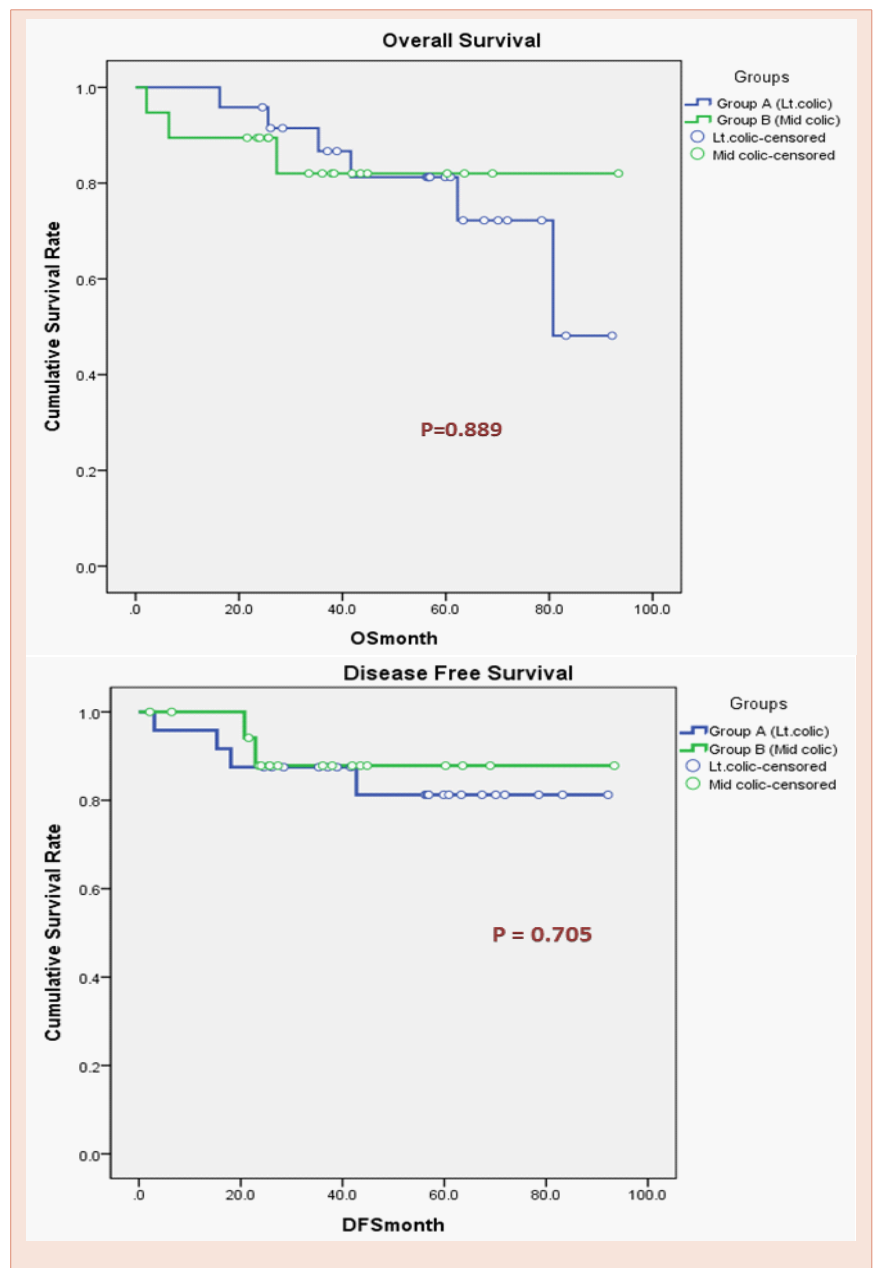

Figure 1: Kaplan Meier Survival Curve. Overall and Disease Free Survival, DFS analyzed at 79.1 month vs. 84.7 month with $95 \%$ confident interval at $(67.4-90.8)$ in group A and $(73.4-96)$ group B respectively. Overall survival rated at 75.2 month vs. 79.1 month with $95 \%$ confident interval $(64.1-86.24)$ and (64.3 -93.9) respectively. could follow ileocolic artery in SFC but it had been rarely reported, hence then, left colectomy stated as the standard procedure of choice in SFC [2]. Left colectomy is therefore increasing in popularity as the uptake of knowledge about feeding vessel increased however variation in practice with regards to the ligation of vascular pedicle root is still remained in controversy.

However, more controversial issue is in how to determine the optimum level of vessel ligation in case of SFC. Indeed, this question has not been proposed earlier and the level of arterial division still till now completely based on surgeon preferences. As long as I know this is the first study to investigate the optimum level of LMA division in SFC.

Essential point to consider during division of feeding vessel to splenic flexure area is the anatomy network of the blood supply to the splenic flexure as it is variable from person to another. It has studied earlier by Griffith et al. [15], demonstrated anatomical description of colon blood supply in 100 cadavers, revealed that splenic flexure colon supplied by LCA in $89 \%$ of cases and $11 \%$ by MCA. Well known knowledge that lympahtic spread will follow arterial system in their drainage [16]. Therefore, SFC carcinoma has dual lymphatic drainage: to the superior mesenteric vessel and to the inferior mesenteric vessels. Particular point of interest, late presentation of SFC could have metastatic lymph nodes in all feeding vessel to splenic flexure area including MCA and LCA at which better prognosis theoretically will be anticipated if division has considered for both vessels at the root, however clinically has not addressed well yet. As opposed to feeding vessels and lymphatic drainage to SFC, there is a direct lymphatic drainage to the splenic hilum As well as to pancreas tail [17], but there was no significant different in 5-year survival if splenectomy and distal pancreatectomy were performed in SFC patients [18]. Lymph node metastasis in splenic hilum has been studied before without existence of malignant cells in splenic hilum, herein, splenectomy has no more considered an important part of SFC management unless if the tumor invading splenic hilum or a consequence bleeding following the tumor excision $[18,19]$.

Furthermore, SFC is rarely seen in CRC, which contributed in lack of literatures resources. In the other hand, location of the tumor in colonic flexures is adding further strain to obtain proper lymphadenectomy as well as in delayed presentation, which both factors attributed in further worsen oncology outcome and patients prognosis. Benedix et al. [20], conducted a multi-centered observational study in 29,658 with colon cancer, showed delayed presentation in the majority of caecum cancer and SFC, particularly at stage III and IV as well as rise in the incidence oflymphatic invasion in SFC. Moreover, Kim et al. [2], stated that, anatomical location of splenic flexure associated with the highest risk of obstruction (49\%) that conducted in the Large Bowel Cancer Project in 4292 patients involved in this study. Therefore, we try to explore and investigate level of vessel ligation in SFC, which might participate in enhancement of overall survival.

From our own experience, we enrolled 43 patients with SFC without any discrimination in patient's demographic data or tumor characteristics details. We considered high vessel ligation to include LMA in 19 patients (44.2\%), however we could not determine oncological benefits over division of LCA plus MMA. DFS analyzed 
at 79.1 month vs. 84.7 month and overall survival rated at 75.2 month vs. 79.1 month respectively, which haven't shown statistical significant between both study arms $(p>0.05)$. However, in study group $\mathrm{A}$, recurrence rate was potentially lesser than study group $\mathrm{B}$, which might affect patients quality of life, hospitality and cost of hospital stay compared to study group $B(p=0.56)$. Although we found no strong direct clinical Impact between two levels of vessel ligation but still randomized clinical trials has to address this regards to emphasize the proper level of vessel ligation in term of oncology safety and patients overall survival.

\section{Limitation}

The main limitation of our study, along with most others, was the small number of patients analyzed. Nevertheless, the data show there were no overall survival differences between study groups but has pointed out potential risk of local recurrence although it was not significant. Another limitation of our study is the retrospective nature, although all procedures, complications and follow up details are coded in our institution and retained in the electronic notes. Therefore we could access all relevant aspects of patient details including procedure type and management. On top of that, involvement of highly experts surgeons in a such large volume cancer center could contributed in yielding higher number of lymph node to an extent might be difficult to discriminate between both groups in term of oncology safety and patients overall outcome. Our study ensured that consideration of vessel ligation in cancer patients is mandatory and further randomized, blinded controlled trials are required to address the ongoing debate in order to determine the optimum level of vessel ligation in patients with SFC.

\section{Conclusion}

In our study, the influence of further vessel ligation (LMA) hasn't shown obvious beneficial effect on overall survival, however has a potential to reduce the risk of local recurrence. The optimal method of vessel ligation level in SFC remains a matter of debate. Randomized controlled trials are required to address this point in near future.

\section{Disclosure of Interest}

Drs. Mahdi H Al Bandar, MD, Yoon Dae Han, MD, Jamal Al Sabilah, MD, Min Soo Cho, MD, HyukHur,MD, Byung Soh Min, $\mathrm{MD}$,Kang Young Lee, MD, and Nam Kyu Kim, MD have no conflicts of interest or financial ties to disclose.

\section{References}

1. Alexander JS, Ganta VC, Jordan PA, Witte MH (2010) Gastrointestina Lymphatics in Health and Disease. Pathophysiology 17: 315-335.

2. Benedix F, Schmidt U, Mroczkowski P, Gastinger I, Lippert H, et al. (2011) Colon Carcinoma-Classification into Right and Left Sided Cancer or According to Colonic Subsite?--Analysis of 29,568 Patients. Eur J Surg Oncol 37: 134139.

3. Bokey EL, Chapuis PH, Dent OF, Mander BJ, Bissett IP, et al. (2003) Surgical Technique and Survival in Patients Having a Curative Resection for Colon Cancer. Dis Colon Rectum 46: 860-866.

4. Derwinger K, Gustavsson B (2011) Variations in Demography and Prognosis by Colon Cancer Location. Anticancer Res 31: 2347-2350.

5. Feng B, Sun J, Ling TL, Lu AG, Wang ML, et al. (2012) Laparoscopic Complete Mesocolic Excision (Cme) with Medial Access for Right-Hemi Colon Cancer: Feasibility and Technical Strategies. Surg Endosc 26: 36693675.

6. Galizia G, Lieto E, De Vita F, Ferraraccio F, Zamboli A, et al. (2014) Is Complete Mesocolic Excision with Central Vascular Ligation Safe and Effective in the Surgical Treatment of Right-Sided Colon Cancers? A Prospective Study. Int J Colorectal Dis 29: 89-97.

7. Griffiths JD (1956) Surgical Anatomy of the Blood Supply of the Distal Colon. Ann R Coll Surg Engl 19: 241-256.

8. Hohenberger W, Weber K, Matzel K, Papadopoulos T, Merkel S (2009) Standardized Surgery for Colonic Cancer: Complete Mesocolic Excision and Central Ligation--Technical Notes and Outcome. Colorectal Dis 11: 354-364.

9. Jamieson JK, Dobson JF (1909) VII. Lymphatics of the Colon: With Special Reference to the Operative Treatment of Cancer of the Colon. Ann Surg 50: 1077-1090.

10. Khafagy MM, Stearns MW, Jr (1973) Carcinoma of the Splenic Flexure. Dis Colon Rectum 16: 504-507.

11. Kim CW, Shin US, Yu CS, Kim JC (2010) Clinicopathologic Characteristics, Surgical Treatment and Outcomes for Splenic Flexure Colon Cancer. Cancer Res Treat 42: 69-76.

12. Levien DH, Gibbons S, Begos D, Byrne DW (1991) Survival after Resection of Carcinoma of the Splenic Flexure. Dis Colon Rectum 34: 401-403.

13. Miyajima N, Yamakawa T (1997) Present State and Problems of Laparoscopic Surgery for Colon and Rectal Carcinoma. Nihon Geka Gakkai Zasshi 98: 380 384.

14. Nakagoe T, Sawai T, Tsuji T, Jibiki M, Ohbatake M, et al. (2001) Surgical Treatment and Subsequent Outcome of Patients with Carcinoma of the Splenic Flexure. Surg Today 31: 204-209.

15. Okuno K (2007) Surgical Treatment for Digestive Cancer. Current Issues Colon Cancer. Dig Surg 24: 108-114.

16. Sadler GP, Gupta R, Foster ME (1992) Carcinoma of the Splenic Flexure--a Case for Extended Right Hemicolectomy? Postgrad Med J 68: 487.

17. Steffen CE, Bokey L, Chapuis PH (1987) Carcinoma of the Splenic Flexure. Dis Colon Rectum 30: 872-874.

18. Toyota S, Ohta H, Anazawa S (1995) Rationale for Extent of Lymph Node Dissection for Right Colon Cancer. Dis Colon Rectum 38: 705-711.

19. Walfisch S, Stern H (1989) Use of Thoracoabdominal Incision for Cancer of the Splenic Flexure in the Obese Patient. Dis Colon Rectum 32: 169-170.

20. Watanabe T, Itabashi M, Shimada Y, Tanaka S, Ito Y, et al. (2012) Japanese Society for Cancer of the Colon and Rectum (Jsccr) Guidelines 2010 for the Treatment of Colorectal Cancer. Int J Clin Oncol 17: 1-29.

Copyright: (C) 2016 Al Bandar MH, et al. This is an open-access article distributed under the terms of the Creative Commons Attribution License, which permits unrestricted use, distribution, and reproduction in any medium, provided the original author and source are credited. 